\title{
Medical disclosure and Covid-19 vaccination
}

\section{Opinion}

Humanity is suffering from not only a pandemic of quasiapocalyptic implications, but also from epic mass confusion and doubts regarding medical advice. It is perfectly understandable but most distressing to behold. Medical history is being written and rewritten every other week and it must be most soul destroying for the public to behold medical experts bereft of the usual authority and confidence. It is also understandable that instead of appreciating the near miraculous roll-out of vaccines designed and produced virtually overnight, man is at serious odds with himself and others as to whether he or she ought to have such a vaccine and at what immediate and long-term cost.

It is most justifiable that uncertainty, doubt, fear and even utter confusion constitute the chief protagonists of the Covid 19 saga. Not only are the goal-posts being moved but in some cases they are being completely annihilated. Thus, this is the first in many people's living memory that no sooner is a vaccine available than it is partly neutered by living mutation of the offending virus. It is of small consolation that such pathogenic mutation is a daily business. Another major dilemma is the very choice of vaccine, even if in reality one must get what one is offered and often consider oneself lucky for getting it.

The Oxford-AstraZeneca Covid 19 Vaccine is perhaps the most currently controversial of vaccines. Some are refusing to take it outright, others take it with much trepidation and a third group seem to accept medical reassurance and take it without qualm. There are not only individual differences in opinion but there also seems to be influence through various groups one might appertain to, regional differences and even national opinions with the French apparently being rather sceptic of vaccines in general.

Keeping in mind the principles of modern disclosure it is a most worthwhile exercise to consider what the law expects from the physician advising a patient on such vaccination and here the Covid-19 AstraZeneca Vaccine is being chosen. At this point it may be healthy to point out that the lay public is desperate for advice from any source considered to have some kind of "inside" information. This of course is sheer nonsense, but it is a fact that doctors, nurses, pharmacists, para-medics and indeed anyone known to be on some healthcare platform is often asked for information and advice. The approached individual may have no knowledge at all but he/she must realise that their answer caries responsibility for it is weighted. Each person put off vaccination is a blow to the crucial time factor race to establish herd immunity.

One aspect which tends to accentuate the weakness of all current scientific argument and advice, is the fact that all Covid-19 knowledge is relative, recent and subject to change as new scientific information becomes available. As one patient put it "You doctors don't know what's what and keep changing your story!" To some extent that's an undeniable fact. And neither should one reject the fact that Medicine has been dealing with an unexpected challenge. In fact let us see what should be disclosed in advising patients regarding the OxfordAstraZeneca Covid-19 Vaccine.

Firstly, one should clearly state that Medicine was obviously not prepared for the new infectious disease known as Cov-19 which is

\author{
Volume 5 Issue 2 - 202I
}

\author{
George Gregory Buttigieg,' Kirill Micallef- \\ Stafrace, ${ }^{2}$ Nicola Micallef-Stafrace ${ }^{3}$ \\ 'Professor, Obstetrics and Gynaecolotgy, University of Malta, \\ Malta \\ ${ }^{2}$ Consultant Department of Orthopaedics, Trauma and Sports \\ Medicine, Mater Dei Hospital Malta \\ ${ }^{3}$ Family Medicine Specialist, Community practitioner with special \\ interest in Aesthetic Medicine, Malta
}

Correspondence: George Gregory Buttigieg, Professor, Obstetrics and Gynaecolotgy, University of Malta, Malta, Email george.g.buttigieg@um.edu.mt

Received: March 29, 2021 | Published: April 19, 2021

infective, may be fatal or seriously damage health. In spite of this lack of preparation medical science has managed to put forward a number of Covid-19 Vaccines. The Oxford-AstraZeneca Cov-19 Vaccine is one such vaccine which is indicated for Covid-19 infections caused by the SARS-CoV2 in individuals 18 years of age and older. These vaccines have become available to the public at a much accelerated rate than usual, not because the usual investigations and tests have been by-passed but rather time-telescoped. This must be stressed and at this point one should discuss the efficacy of the vaccine as revealed by the latest studies and any information relating to efficiency as well as the latest on the disease variants. The patient must also be warned that once vaccinated the full preventive measures including masking and social distancing were not to be abandoned.

\section{The situation is indeed complex because}

1. Covid-19 virus itself is evolving to ensure its survival leading to such variants as the UK, South African, Brazilian and Indian variants. These variants can occur de novo anywhere else at any time. The chance of these variants appearing would be diminished if the actual number of infectious cases is diminished. This is one advantage of mass vaccination which may not be obvious and should be pointed out.

2. Updating of scientific information regarding the disease, its evolving variants as well as its vaccines is constant and is likely to lower medical confidence in the public. This is not scientific confusion but the very opposite. It may well be for example, that the present system of giving a vaccine may be modified or enhanced by a vaccine booster being given say every 6 or 9 or 12 months.

3. New side effects related to AstraZeneca vaccine and any other may only emerge with time. This is one issue which may worry patients most and which may keep them from being vaccinated. 
One must stress that: As the situation stands, it is far less risky to be vaccinated now than not, on the grounds of what other side effects may emerge later.

The physician is also duty bound to stress that when bodies such as the European Medicines Agency have cleared a vaccine or indeed any other drug for human use, their scientific scrutiny in safeguarding the lives and health of the populations for whom the vaccine/medicine is intended, is extremely intense and based on much past experience.

With regards to the vaccine in question and thromboembolic events the physician must remember that cases have been reported, albeit very rarely, leading to local suspension of specific batches of Oxford-AstraZeneca vaccine and even to the vaccine itself. Some cases have even had a fatal outcome. These complications involve the occurrence of a combination of thrombosis or thrombo-embolism and thrombocytopenia leading to haemorrhage. Unusual sites havebeen the targets of such pathology resulting in cerebral venous sinus thrombosis, mesenteric vein thrombosis and also arterial thrombosis. The majority of these very rare cases occurred in the first 7-14 days post-vaccination and have occurred in women under 55 years of age.

The Pharmacovigilance Risk Assessment Committee (PRAC) of the European Medicines Agency has investigated the situation fully and has recommended both an update to the product information to reflect the current knowledge as well as stressed to physicians the need to continue reporting adverse reactions from the involved vaccine.

Modern principles of disclosure demand that the facts are disclosed to the patient but in the correct light as science interprets them. Thus, the patient must know about the rare occurrence of development of thrombosis and thrombocytopenia but also that the risk is so low that the advantages of taking the vaccine outstrip by far the risks. The obligation holds to warn the patient of the potential (however rare) side-effects with the scope that however rare, any such pathology is caught and managed as early as possible. Hence the patient must be alerted to report any painful and/or swollen leg, shortness of breath or persistent abdominal pain. In the rare case of thrombosis affecting the central nervous system the patient may develop and hence must report immediately any severe or persistent headaches and/or blurred vision. In case of the development of thrombocytopenia, the patient may present with skin bruising (apart from the site of the vaccination injection) within a few days of being vaccinated.

Modern medical ethical and legal requirements which demand that patients know the facts to enable them to choose/refuse their own treatment. This simply cannot be put aside by any argument which the physician may think is valid in his mind. The medico-legal implications of Covid-19, its vaccination and the short and long-term sequelae to the disease and its treatment, will no doubt emerge in time. However, apart from medico-legal considerations, patients appreciate when they are not treated liken children and are given the full but correct facts as they stand and in this situation with the proviso that tomorrow may bring more knowledge. Neither the complexity of the issue, nor its malleability, neither the importance of herd immunity nor a physician "knowing better", may excuse the dereliction of duty of full and complete disclosure in a simplified but correct form. Sooner rather than later the concept will, no doubt, make its presence felt in a Court of law.

\section{Acknowledgments}

None.

\section{Conflicts of interest}

The author declares there is no conflict of interest. 